\title{
Aspectos fisiopatológicos da hipertensão arterial dependente de angiotensina II: revisão integrada da literatura
}

\author{
Natália Tabosa Machado Calzerraa, Camila Figueiredo Gomesª, Thyago Moreira de Queiroz ${ }^{\mathrm{b} *}$ \\ a Instituto de Educação Superior da Paraíba - IESP, João Pessoa, Paraíba, Brasil. \\ b Departamento de Fisiologia e Farmacologia, Universidade Federal do Ceará, Fortaleza, 60430-275, Brasil. \\ *thyagoqueiroz@gmail.com
}

Recebido: 25 janeiro 2018 / Aceito: 23 abril 2018 / Publicado online: 28 maio 2018

\begin{abstract}
Resumo
A hipertensão arterial é uma das principais causas de morte em países desenvolvidos e em desenvolvimento, causando um grande impacto na saúde humana. $\mathrm{O}$ estresse oxidativo tem sido apontado como um mecanismo fundamental na hipertensão arterial dependente de angiotensina II, uma vez que modula a função barorreflexa em vários processos de fisiopatológicos. O presente trabalho teve como objetivo a realização de uma revisão de literatura abordando os recentes mecanismos que promovem alterações da hipertensão arterial dependente de angiotensina II. Foram enfatizados dois modelos dependentes de angiotensina II: dois-rins-um-clipe (2R1C) e desoxicorticosterona (DOCA-sal). A pesquisa corresponde a uma revisão bibliográfica de caráter descritivo, baseada na literatura científica atual, pesquisada em várias bases de dados. Estudos corroboram a hipótese que a angiotensina II é um dos principais agentes envolvidos no desenvolvimento da hipertensão arterial e que o aumento desse peptídeo é multifatorial, podendo induzir, em especial, o aumento do estresse oxidativo que, por sua vez, promove alterações em uma metaloprotease 17 (ADAM17) e disfunções na enzima conversora da angiotensina 2 (ECA2). Assim, novas ferramentas que induzam a diminuição do estresse oxidativo promovem efeitos benéficos, constituindo novos alvos terapêuticos na prevenção e tratamento de hipertensão arterial.
\end{abstract}

Palavras-chave: Hipertensão, Angiotensina II, ECA 2, estresse oxidativo.

\section{Physiological aspects of angiotensin II-dependent hypertension: an overview}

\begin{abstract}
Hypertension is one of the main causes of death in developed and developing countries, inducing great impact in human health. Oxidative stress has been implicated as a key mechanism in Angiotensin II-dependent hypertension, since it modulates the baroreflex function in many pathophysiological processes. This study aimed to conduct a literature review addressing the recent mechanisms that promote changes in Angiotensin II-dependent hypertension. Here we highlighted two Angiotensin IIdependent hypertension models: two kidney-one-clip (2K1C) and deoxycorticosterone (DOCA-salt) hypertension. Bases on latest studies presented in important data base, it is possible to suggest that Angiotensin II is one of the main agents in hypertension development and the increase of this peptide is derived by multiple causes, which, in particular, acts as an inductor in oxidative stress production, in turn, it induces changes in a metalloprotease 17 (ADAM-17) and disruption of ACE2 compensatory activity. Thus, new tools that induce a reduction of oxidative stress promote beneficial effects, providing novel therapeutic targets to prevent and treat the Angiotensin II-dependent hypertension.
\end{abstract}

Keywords: Hypertension, Angiotensin II, ACE 2, oxidative stress.

\section{Introdução}

A hipertensão arterial (HA) é uma condição clínica de caráter multifatorial, caracterizado por níveis elevados e sustentados de pressão arterial (PA) (PA sistólica $\geq 140$ $\mathrm{mmHg}$ e/ou PA diastólica $\geq 90 \mathrm{mmHg}$ ) (VII Diretriz Brasileira de Hipertensão, 2016). Esta condição tem sido indicada como um fator de risco importante para a morbidade e mortalidade precoces causadas por doenças cardiovasculares (Simão et al., 2008).

Estima-se que $29 \%$ da população adulta do mundo, ou aproximadamente 1,56 bilhão de pessoas, estarão hipertensas em 2025. No Brasil, HA atinge 32,5\% (36 milhões) de indivíduos adultos e mais de $60 \%$ dos idosos, de forma a contribuir direta ou indiretamente para $50 \%$ das mortes por doenças cardiovasculares (Saurabh et al., 2014). Neste cenário, a HA apresenta elevados custos médicos e socioeconômicos decorrentes principalmente das complicações que a acompanha, tais como: acidente vascular cerebral (AVC), doença arterial coronariana, insuficiência cardíaca e insuficiência renal crônica (VII Diretriz Brasileira de Hipertensão, 2016). 
Uma característica da hipertensão é a hiperatividade do sistema renina-angiotensina, que contribui para várias alterações funcionais e estruturais em vários órgãos-alvo. Um dos produtos deste sistema, a angiotensina II (Ang II) promove lesão vascular por induzir vasoconstrição, proliferação e hipertrofia de células musculares lisas e inflamação vascular, bem como a degradação da matriz extracelular. Além disso, contribui com o aumento da contratilidade miocárdica, retenção de sódio e água e elevação da pressão arterial (Santos et al., 2012: Thieme et al., 2017).

Muitos estudos demonstraram que níveis elevados circulantes e teciduais de Ang II são estímulos potentes para aumentar a produção de espécies reativas de oxigênio (ROS) e elevar a expressão de citocinas pró-inflamatórias que ativam ainda mais o estresse oxidativo, contribuindo para o desenvolvimento e manutenção da hipertensão (Silva, 2017). O estresse oxidativo também está associado com a regulação negativa da enzima conversora de angiotensina tipo 2 (ECA2) por meio da ação da desintegrina e metaloprotease 17 (ADAM17) na hipertensão (Queiroz et al., 2015). Além disso, ROS modulam a função barorreflexa, que é geralmente anormal na hipertensão (Loperena e Harrison, 2017).

O presente trabalho teve como objetivo a realização de uma revisão da literatura abordando os recentes mecanismos que promovem alterações da hipertensão arterial dependente de Ang II. Além disso, foram enfatizados, principalmente, dois modelos dependentes de Ang II: dois-rins-um-clip (2R1C) e DOCA-sal.

\section{Material e Métodos}

O presente trabalho trata-se de uma pesquisa bibliográfica acerca das alterações da hipertensão arterial dependente de angiotensina II. Para a elaboração desta revisão foi realizado um levantamento na literatura nacional e internacional utilizando os seguintes bancos de dados Pubmed, Scielo, Google Acadêmico, Science Direct, Sciepub. Os descritores utilizados para pesquisa foram: hipertensão arterial, angiotensina II, ECA2, estresse oxidativo.

Os critérios de inclusão para construção desse trabalho foram artigos originais e de revisão que abordavam as características gerais da hipertensão arterial dependente de angiotensina II, elucidando o envolvimento do estresse oxidativo; que apresentam disponibilidade na forma de texto completo; e que enfatizam os dois modelos animais de hipertensão dependentes de angiotensina II: dois-rins-umclipe (2R1C) e DOCA-sal. Foram excluídos resumos de teses ou dissertações e publicações que não discutiam a relação entre a angiotensina II, estresse oxidativo e hipertensão. As informações foram captadas e analisadas para corresponder a proposta do trabalho.

\section{Resultados e Discussão}

Diversos estudos relacionam a participação do aumento da atividade do sistema nervoso simpático no desenvolvimento da HA (Irigoyen et al., 2005). Adicionalmente, relata-se que o aumento da atividade simpática e a redução da atividade parassimpática estão diretamente relacionados à disfunção do barorreflexo, uma vez que os barorreceptores modulam a atividade autonômica (Botelho-Ono et al., 2011).

Barorreceptores situados na camada adventícia do arco aórtico e seio carotídeo sentem variações na pressão arterial, propagando potenciais de ação para o Sistema Nervoso Central (SNC), que por sua vez desencadeiam ajustes autonômicos reflexos que tamponam as alterações da pressão arterial. Em condições patológicas, como na hipertensão, há prejuízo na regulação autonômica da pressão arterial, resultando em danos na sensibilidade do barorreflexo (Salgado et al., 2009; Siti et al., 2015).

Dentre as regiões do SNC envolvidas no controle reflexo pressão arterial, destacam-se o núcleo paraventricular do hipotálamo (PVN) e o bulbo ventrolateral rostral (RVLM). O primeiro é uma das principais áreas do SNC envolvidas no controle autonômico e neuro-humoral da pressão arterial (Burmeister et al., 2011), já que possui tanto neurônios simpáticos quanto neurônios vasopressinérgicos (Xia et al., 2013). Os estímulos das vias da vasopressina provenientes do PVN contribuem para um aumento da atividade simpática, que por sua vez induz uma elevação da pressão arterial (Colombari et al., 2010). Além disso, a ativação de mecanismos que modulam a formação de Ang II no PVN pode promover alterações na atividade e/ou reflexo simpático, ou ainda na sensibilidade do barorreflexo tornando-se, portanto um órgão de grande interesse na investigação dos mecanismos de alteração da pressão arterial (Burmeister et al., 2011).

O estresse oxidativo modula a função barorreflexa em vários processos fisiopatológicos. Por exemplo, a administração de sequestradores de radicais livres exógenos, tais como a superóxido dismutase e catalase, para o seio carotídeo de coelhos com aterosclerose induzida experimentalmente, aumenta a função barorreflexa (Nishi et al., 2010). Estes resultados sugerem um papel modulatório de espécies reativas de oxigênio sobre barorreceptores carotídeos. Além disso, a infusão intravenosa aguda de ácido ascórbico, um conhecido antioxidante, aumenta a sensibilidade do barorreflexo em pacientes com insuficiência cardíaca (Queiroz et al., 2012)

$\mathrm{O}$ aumento de espécies reativas de oxigêncio tem sido apontado como um mecanismo chave na hipertensão arterial dependente de Ang II. ROS são importantes no aumento da pressão arterial, após administração de Ang II, a nível sistêmico ou diretamente no SNC (Houston, 2005).

Considerando que Ang II é composta por oito aminoácidos, tornando-a incapaz de atravessar a barreira hematoencefálica (BHE), os mecanismos que envolvem as ações da Ang II no cérebro que modulam atividade simpática induzem hipertensão arterial permanecem desconhecidos (Carvalho et al., 2012).

A hipótese mais aceita é a de que Ang II atua sobre neurônios em regiões específicas do cérebro, conhecidas como órgãos circuventriculares (CVOs), como por exemplo o órgão subfornical (SFO), área postrema (AP) e órgão vascular da lâmina terminal (OVLT), que são parcialmente ausentes de barreira hematoencefálica. Como resultado, a ativação dos CVOs induz uma produção local de Ang II em áreas cerebrais protegidas pela BHE, principalmente o RVLM, que por sua vez altera o tônus simpático levando a hipertensão (Carvalho et al., 2012). Outro importante fator é o de que componentes do sistema renina-angiotensina (SRA) estão presentes em várias 
regiões do cérebro que são envolvidas na regulação central da pressão arterial, incluindo o PVN, RVLM e o núcleo do trato solitário (NTS) (Paton et al., 2008). Portanto, nessas regiões há formação local de Ang II e consequente aumento da pressão. Entretanto, evidências mostraram um papel fundamental no estágio inicial da hipertensão arterial. Ang II circulante induz ativação de leucócitos que transmigram a parede vascular, induzem a produção de citocinas próinflamatórias como a IL-1 $\beta$, IL-6 e TNF- $\alpha$, que são conhecidas por modularem a função neuronal, levando a simpatoexcitação (Waki et al., 2011)

$\mathrm{O}$ aumento nos níveis de Ang II tanto em nível sistêmico quanto no SNC pode desencadear um aumento na atividade simpática e aumento da pressão arterial. Este fato foi comprovado por estudos que revelaram a hipertensão arterial induzida por Ang II, como um resultado causado por ambos os efeitos centrais quanto por elevações da PA periféricas. Estes efeitos promovem a ativação de células $\mathrm{T}$ e desenvolvimento de inflamação vascular. Esses achados suportam um mecanismo de retroalimentação positiva, no qual pequenas elevações da pressão arterial induzem ativação de células $\mathrm{T}$, que por sua vez, desencadeiam inflamação e aumento da pressão arterial, levando a uma hipertensão arterial severa (Marvar et al., 2010). De acordo com recente estudo desenvolvido por Biancardi et al. 2014, pôde-se notar que a Ang II, via ativação de receptores para Ang II tipo 1 (AT1R), induz um efeito inflamatório deletério sobre a BHE, promovendo o rompimento dessa barreira física, e assim permitindo o acesso hormônico em áreas do SNC normalmente protegidas, como PVN, RVLM e o NTS.

A produção de Ang II é decorrente da ativação do SRA. Uma vez ativo, este sistema consiste em uma cascata enzimática formada de duas etapas, catalisadas pela renina e pala enzima conversora de angiotensina (ECA). A renina atuará na conversão do angiotensinogênio em um decapeptídeo, a angiotensina I. Este polipeptídeo apresenta características bastante instáveis, sendo rapidamente convertido por ação da ECA em Ang II (Abadir, 2011).

Uma proteína recentemente identificada, e considerada um regulador negativo do SRA, a ECA2, cliva Ang I e Ang II em Ang 1-9 e Ang 1-7, respectivamente (Chang et al., 2011). Estudos mostram que a ECA2 está envolvida em mediar efeitos como a natriurese, vasodilatação e inibição da insuficiência cardíaca. Estes efeitos parecem estar relacionados com a redução do estresse oxidativo, uma vez que a deficiência de ECA2 induz um aumento de Ang II e ROS, mediado pela ativação do sistema NADPH oxidase, com exacerbação do estresse oxidativo, levando a hipertensão (Chang et al., 2011; Xiao et al., 2011; Rabelo et al., 2016).

A primeira demonstração de uma ação biológica para a Ang 1-7 foi realizada por Schiavone et al. (1988) (Santos, Campgnole-Santos, Andrade, 2000). Ang 1-7 exibe um efeito antiarrítmico, protege o coração de lesões induzidas por isquemia/reperfusão e preserva a função do ventrículo esquerdo após infarto do miocárdio induzida por isoproterenol (Loot et al., 2002). Além disso, Grobe et al. (2007) mostraram que 28 dias de infusão intravenosa de Ang 1-7 atenua a fibrose cardíaca induzida por DOCA-sal ou hipertensão induzida por Ang II, sem alterar o grau de hipertrofia cardíaca ou pressão arterial basal. A maior parte das ações induzidas por Ang 1-7 é medida por seu receptor "Mas", acoplado a proteína G (Santiago et al., 2010), com consequente diminuição da pressão arterial. Adicionalmente, Ang 1-7 foi capaz de aumentar a sensibilidade do barorreflexo (Santos, 2014).

A ECA2, que foi descoberta no ano 2000, é um exemplo de proteína integral de membrana, com um extenso domínio catalítico, uma simples hélice transmembrana e um domínio carboxiterminal curto. A ECA2 pode sofrer shedding por liberar o ectodomínio cataliticamente ativo da superfície celular para o meio extracelular (Lai et al., 2011).

Um dos principais grupos das proteases sheddases é a família ADAM (A Desintegrim And Metalloproteinase). ADAM17 foi considerada primariamente, a responsável pela liberação da forma solúvel da ECA2, por proporcionar o shedding dessa enzima. Dessa maneira, a ECA2 deixará de mediar a conversão de Ang II em Ang 1-7, reduzindo os níveis membranares desta enzima e aumentando sua forma solúvel, com perda de sua atividade compensatória sobre pressão arterial (Xia et al., 2013).

Alterações nas cascatas de sinalização supracitadas podem desencadear quadros de hipertensão arterial dependentes de Ang II. A hipertensão renovascular do tipo 2R1C é um modelo de hipertensão induzido cirurgicamente pela contrição da artéria renal, induzindo a isquemia renal e consequentemente ativando um dos sistemas vasoconstritores mais potentes do organismo, o Sistema Renina-angiotensina (SRA) (PrietoCarrasquero et al., 2008; Ferreira, 2017).

DOCA-sal é um modelo de hipertensão com baixos níveis de renina, onde há a administração de um mineralocorticoide, desoxicorticosterona, em animais uni-nefrectomizados e com suplementação de $1 \%$ de $\mathrm{NaCl}$. Foi demonstrado que é necessário o tratamento conjunto do excesso diário de sal e do esteroide para desenvolvimento da hipertensão DOCA-sal, já que somente o tratamento com DOCA ou apenas o consumo excessivo de sal não é capaz de proporcionar uma elevação da pressão arterial. Nesse tipo de hipertensão há uma supressão do SRA periférico, porém há uma supra regulação do SRA a nível do SNC (Grobe et al., 2011; Chrissobolis, 2017).

Apesar das diferenças apresentadas nos modelos de hipertensão supracitados, e das vantagens que são inerentes a cada modelo, ambos possuem, pelo menos, um fator em comum que é o aumento dos níveis de Ang II. Além disso, são de fácil execução e muito eficientes no estudo da hipertensão arterial.

Durante a infusão periférica de Ang II, bem como no modelo de hipertensão DOCA-sal, foi observado que citocinas pró-inflamatórias, incluindo TNF- $\alpha$, IL-1 $\beta$, IL-6 e a quimiocina MCP-1 apresentavam-se elevadas no PVN. Esses estudos corroboram a hipótese da hipertensão arterial como uma condição inflamatória. Devido ao interessante papel dos fatores imunológicos na regulação da hipertensão arterial, bem como na participação do SNC no controle da pressão arterial. Essas hipóteses ressaltam a importância da conexão neuroimune-fisiológica no processo da hipertensão arterial (Xia et al., 2013).

A importância da avaliação dos efeitos no hipotálamo devese ao fundamental papel desta área do SNC no controle da hipertensão arterial mediado pelo estresse oxidativo e Ang II. 
Por exemplo, o reflexo simpático cardíaco mostrou-se estar envolvido na formação de ROS mediada por Ang II no PVN (Zhang et al., 2006). Além disso, efeitos pressóricos centrais e sistêmicos induzidos por Ang II estão conectados com a formação de ROS mediada por NADPH oxidase no PVN (Burmeister et al., 2011).

\section{Conclusões}

A partir dos estudos levantados na presente revisão, permite-se sugerir que a Ang II é um dos principais agentes no desenvolvimento da hipertensão arterial e que o aumento desse peptídeo é multifatorial, podendo induzir, em especial, um aumento do estresse oxidativo, que por sua vez, promove alterações em uma metaloprotease 17 (ADAM17) e disfunção na ECA2. Assim, novas ferramentas que induzam a diminuição do estresse oxidativo promovem efeitos benéficos, constituindo novos alvos terapêuticos na prevenção e tratamento da hipertensão arterial.

\section{Referências}

Abadir, P.M. 2011. The frail renin-angiotensin system. Clinics in Geriatrics Medicine, 27(1): 3-65.

Biancardi, V.C.; Son, S.J.; Ahmadi, S.; Filosa, J.A.; Stern, J.E. 2014. Circulating angiotensin II gains access to the hypothalamus and brain stem during hypertension via breakdown of the blood-brain barrier. Hypertension. 63(3): 572-9.

Botelho-Ono, M.S.; Pina, H.V.; Sousa, K.H.F.; Nunes, F.C.; Medeiros, I.A.; Braga, V.A. 2011. Acute superoxide scavenging restores depressed baroreflex sensitivity in renovascular hypertensive rats. Autonomic Neuroscince Basic and Clinical. 159(1-2): 28-44.

Burmeister, M.A.; Young, C.N.; Braga, V.A.; Butler, S.D.; Sharma, R.V.; Davisson, R.L. 2011. In vivo bioluminescence imaging reveal redoxregulated activator protein-1 activaction paraventricular nucleus of mice with renovascular hypertension. Hypertension. 57:289-297.

Carvalho, A.S.; Guimaraes, D.D.; Dantas, B.P.V.; Carreiro, J.N.; MendesJunior, L.G.; França-Silva, M.S.; Monteiro, M.M.O.; Alves, N.F.B.; Porpina, S.K.P.; Queiroz, T.M.; Braga, V.A. 2012. Brain AngiotensinII-derived Reactive Oxygen Species: Implications for High Blood Pressure. Journal of Hypertension.

Chang, S.Y.; Chen, Y.W.; Chenier, I.; Tran sle, M.; Zhang, S.L. 2011. Angiotensin II type II receptor deficiency accelerates the development of nephropathy in type I diabetes via oxidative stress and ACE2. Experimental Diabetes Research. 2011: 1-12.

Chrissobolis, S.; Dinh, Q.N.; Drummond, G.R.; Sobey, C.G. 2017. Role of Oxidative Stress in Hypertension. In: Studies on Atherosclerosis. Springer US: 59-78.

Colombari, E.; Colombari, D.S.; Li, H.; Shi, P.; Dong, Y.; Jiang, N.; Raizada, M.K.; Sumners, C.; Murphy, D.; Paton, J.F. 2010. Macrophage migration inhibitory factor in the paraventricular nucleus plays a major role in sympathoexcitatory response to salt. Hypertension, 53(5): 95663.

Ferreira, L.G.; Evora, P.R.B.; Capellini, V.K.; Albuquerque, A.A.; Carvalho, M.T.M.; Gomes, R.A.S.; Parolini, M.T.; Celotto, A.C. 2017. Effect of rosmarinic acid on the arterial blood pressure in normotensive and hypertensive rats. Role of Ace. Phytomedicine. 38: 158-165.

Grobe, J.L.; Buehrer, B.A.; Hilzendeger, A.M.; Liu, X., Davis, D.R.; Xu, D.; Sigmund, C.D. 2011. Angiotensinergic signaling in the brain mediates metabolic effects of deoxycorticosterone (DOCA)-salt in C57 mice. Hypertension. 57(3): 600-607.

Grobe, J.L.; Mecca, A.P.; Lingis, M.; Shenoy, V.; Bolton, T.A.; Machado, J.M. 2007. Prevention of angiotensin II-infuced cardiac remodeling by angiotensin (1-7). American Hournal of Physiology Heart and Circulatory Physiology. 292(2): H742.

Houston, M.C. 2005. Nutraceuticals, vitamins, antioxidants and mineralz in the prevetion and treatment of hypertension. Progress in Cardiovascular Diseases. 47(6): 396-449.
Irigoyen, M.C.; Fiorino, P.; Angelis, K.; Krieger, E.M. 2005. Sistema nervoso simpático e hipertensão arterial: reflexos cardiovasculares. Revista Brasileira de Hipertensão. 12(4): 229-233.

Lai, Z.W.; Hanchapola, I.; Steer, D.L.; Smith, A.I. 2011. AngiotensinConverting Enzyme 2 ectodomain Shedding Cleavage-Site Identification: Determinants and Constraints. Biochemistry. 50(23): 5182-5194.

Loot, A.E.; Roks, A.J.M.; Henning, R.H.; Tio, R.A.; Suurmeijer, A.J.H.; Boomsma, F.; Gilst, W.H. 2002. Angiotensin (1-7) Attenuates the development of heart failure after myocardial infarction in rats. Circulation. 105(13): 1548-1550.

Loperena, R.; Harrison, D.G. 2017. Oxidative stress and hypertensive diseases. Medical Clinics. 101(1): 169-193.

Marvar, P.J.; Thabet, S.R.; Guzik, T.J.; Lob, H.E.; Mccann, L.A.; Weyand, C.; Gordon, F.J.; Harrison, D.G. 2010. Central and peripheral mechanisms of T-lymphocyte activation and vascular inflammation produced by angiotensin II-induced hypertension. Circulation Research. 107(2): 263270.

Nishi, E.E.; Oliveira-Sales, E.B.; Bergamaschi, C.T.; Oliveira, T.G.; Boim, M.A.; Campos. R.R. 2010. Chronic antioxidante treatment improves arterial renovascular hypertension and oxidative stress markers in the kidney in Wistar rats. American Journal of Hypertension. 23: 473-480.

Paton, J.F.R.; Wang, S.; Polson, J.W.; Kasparov, S. 2008. Signalling across the blood brain barrier by angiotensin II: novel implications for neurogenic hypertension. Journal of Molecular Medicine. 86(6): 705-710.

Prieto-Carrasquero, M.C.; Botros, F.T.; Pagan, J.; Kobori, H.; Seth, D.M.; Casarini, D.E.; Navar, L.G. 2008. Collecting duct renin is upregulated in both kdneys of 2-kidney, 1-clip Goldblatt hypertensive rats. Hypertension. 51(6): 1590-1596.

Queiroz, T.M.; Giumarães, D.D.; Mendes-Junior, L.G.; Braga, V.A. 2012. $\alpha$ Lipoic Acid Reduces Hypertension and Increases Baroreflex Sensitivity in Renovascular Hypertensive Rats. Molecules. 17(11): 13357-13367.

Queiroz, T.M.; Xia, H.; Filipeanu, C.M.; Braga, V.A.; Lazartigues, E. 2015. $\alpha$ Lipoic acid reduces neurogenic hypertension by blunting oxidative stressmediated increase in ADAM17. American Journal of Physiology Heart and Circulatory Physiology. 309(5): 926-934.

Rabelo, L.A.; Todiras, M.; Nunes-Souza, V.; Qadri, F.; Szijártó, I.A.; Gollasch, M.; Penninger, J.F.; Baeder, M.; Santos, R.A.; Alenia, N. 2016. Genetic deletion of ACE2 induces vascular dysfunction in C57BL/6 mice: role of nitric oxide imbalance and oxidative stress. PloS one. 11(4): 1-16.

Salgado, M.C.; Justo, S.V.; Joaquim, L.F.; Fazan, R.Jr.; Salgado, H.C. 2009. Role of nitric oxide and prostanoids in attenuation of rapid baroreceptor resetting. American Journal of Physiology Heart and Circulatory Physiology. 290(3): 1059-1063.

Santiago, N.M.; Guimarães, P.S.; Sirvente, R.A.; Oliveira, L.A.; Irigoyen, M.C.; Santos, R.A.; Campagnole-Santos, M.J. 2010. Lifetime overproduction of circulating Angiotensin (1-7) attenuates deoxycorticosterone acetate-salt hypertension-induced cardiac dysfunction and remodeling. Hypertension, 55(4): 889-96.

Santos, P.C.J.L.; Krieger, J.E.; Pereira, A.C. 2012. Renin-angiotensin system, hypertension, and chronic kidney disease: pharmacogenetic implications. Journal of pharmacological sciences. 120(2): 77-88.

Santos, R.A. 2014. Angiotensin (1-7). Hypertension. 63(6): 1138-47.

Saurabh, R.S.; Prateek, S.S.; Jegadeesh, R. 2014. The determinants and scope of public health interventions to tackle the global problem of hypertension: A review. International Journal of Preventive Medicine. 5 (7): 807-812.

Schiavone, M.T.; Santos, R.A.S.; Brosnihan, K.B.; Khosla, M.C.; Ferrario, C.M. 1988. Release of vasopressin from the rat hypothalamoneurohypophysial system by angiotensin (1-7) heptapeptide. Proceedings of the National Academy of Sciences USA. 85(11): 4095-8.

Simão, M.; Hayashida, M.; Santos, C.B.; Cesarino, E. J.; Nogueira, M.S. 2008. Hipertensão Arterial Entre Universitários da Cidade de Lubango, Angola. Revista Latino Americana de Enfermagem. 16(4).

Sebastião, D.; Silva Jr.; Jara, Z.P.; Peres, R.; Lima, L.S.; Scavone, C.; Montezano, A.C.; Touys, R.M.; Casarini, D.E.; Michelini, L.C. 2017. Temporal changes in cardiac oxidative stress, inflammation and remodeling induced by exercise in hypertension: Role for local angiotensin II reduction. PloS one. 12(12): e0189535.

Silva Jr, S.D.; Jara, Z.P.; Peres, R.; Lima, L.S.; Scavone, C.; Montezano, A.C.; Touyz, R. M.; Casarini, D.E.; Michelini, L.C. 2017. Temporal changes in cardiac oxidative stress, inflammation and remodeling induced by exercise 
in hypertension: Role for local angiotensin II reduction. PloS one. 12(12): e0189535, 2017.

Siti, Hawa N.; Kamisah, Y.; Kamsiah, J. 2015. The role of oxidative stress, antioxidants and vascular inflammation in cardiovascular disease (a review). Vascular pharmacology. 71: 40-56.

Thieme, M.; Sivritas S.H.; Mergia E.; Potthoff S.A.; Guang Yang G.; Hering L.; Grave K.; Hoch H.; Rump L.C.; Stegbaue J. 2017. Phosphodiesterase 5 inhibition ameliorates angiotensin II-dependent hypertension and renal vascular dysfunction. American Journal of Physiology-Renal Physiology. 12(3): F474-F481.

VII Diretriz Brasileira de Hipertensão Arterial. 2016. Arquivos Brasileiros de Cardiologia. 107(3).

Waki, H.; Gouraud, S.S.; Maeda, M.; Raizada, M.K.; Paton, J.F. 2011. Contributions of vascular inflammation in the brainstem for neurogenic hypertension. Respiratory Physiology and Neurobiology. 178(3): 422428.

Xia, H.; Sriramula, S.; Chhabra, K.H.; Lazartigues, E. 2013. Brain angiotensin-converting enzyme type 2 shedding contributes to the development of neurogenic hypertension. Circulation Research. 113(9): 1087-1096.

Xiao, L.; Gao, L.; Lazartigues, E.; Zucker, I.H. 2011. Brain-Selective Overexpression of Angiotensin-Converting Enzyme 2 Attenuates Sympathetic Nerve Activity and Enhances Baroreflex Fuction In Chronic Heart failure. Hypertension. 58: 1057-1065.

Zhang, Y.; Yu, Y.; Zhang, F.; Zhong, M.K.; Shi, Z.; Gao, X.Y.; Want, W.; $\mathrm{Zhu}$, G.Q. 2006. $\mathrm{NAD}(\mathrm{P}) \mathrm{H}$ oxidase in paraventricular nucleus contributed to the effect of angiotensin II cardiac sympathetic afferent reflex. Brain Research. 1082(1): 132-141. 